\title{
Back-to-back substrate wafer bonding: A new approach to the fabrication of double-side coated wafers
}

\author{
P. Kopperschmidt ${ }^{1}$, G. Kästner ${ }^{1}$, D. Hesse ${ }^{1}$, U.M. Gösele ${ }^{1}$, M. Lorenz ${ }^{2}$ \\ ${ }^{1}$ Max-Planck-Institute of Microstructure Physics, Weinberg 2, D-06120 Halle, Germany \\ (Fax: +49-345/5511223, E-mail: kopper@mpi-msp-halle.mpg.de) \\ ${ }^{2}$ Department of Experimental Physics II, University of Leipzig, Linnéstraße 5, D-04103 Leipzig, Germany
}

Received: 9 October 1996 / Accepted: 4 November 1996

\begin{abstract}
We present a novel method for fabrication of double side coated wafers by back-to-back Direct Wafer Bonding (DWB). Two 3 inch sapphire wafers (R-cut) were coated each with $\mathrm{YBa}_{2} \mathrm{Cu}_{3} \mathrm{O}_{7-\delta}$ by laser ablation on one side. The wafers were then directly bonded with their uncoated sides together in a microcleanroom. Subsequent heating increased the bond energy up to energies sufficient for fabrication of hybrid devices working at cryogenic temperatures. TEM cross sections reveal direct contact of the two sapphire lattices.
\end{abstract}

PACS: 68.35.-p; 68.55.-a; 74.72.-h

Conventional design of double side coated films on large sapphire or $\mathrm{LaAlO}_{3}$ substrate wafers are currently fabricated by means of standard epitaxial procedures, e.g. pulsed laser deposition, off-axis sputtering and thermal co-evaporation up to $3^{\prime \prime}, 8^{\prime \prime}$ and $9^{\prime \prime}$ wafer size, respectively. Substrate wafers are coated one after the other by turning them around after the deposition on one side. Hence both films pass through different fabrication cycles, making it difficult to achieve thin films with specific and uniform properties. The fabrication of films with purposely different but specific properties on each face of the wafer is not easily possible by standard deposition methods. Insulating materials with low dielectric losses, double side coated with high temperature superconductors (HTSC), are ideal for filters and resonators in the range of microwave frequencies $[1,2]$.

We present results of a novel technique to fabricate double side coated YBCO films on sapphire wafers by backto-back wafer bonding, as first suggested in ref. [3]. The procedure is schematically illustrated in Fig. 1.

The wafer bonding technique is known as a versatile method to join different materials together without considering their crystallographic orientation, structure, or lattice mismatch [4, 5]. Two mirror polished wafers are brought into contact at room temperature in a microcleanroom. A thermal treatment increases the surface energy up to binding energies of the bulk material.

In our experiment two $400 \mu \mathrm{m}$ thick double side polished sapphire wafers (3", R-cut) with a $\mathrm{CeO}_{2}$ buffer layer were coated with a c-oriented epitaxial YBCO film by Pulsed
A

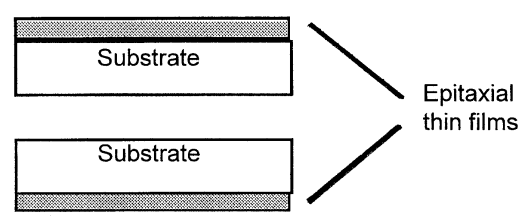

B

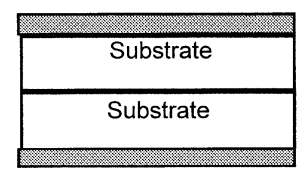

C

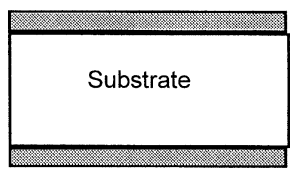

Fig. 1A-C. Fabrication of double side coated wafer by back-to-back wafer bonding. A Two wafers are coated on one side. B The wafers are brought into contact with their uncoated sides. C Annealing yields a two side coated single wafer

Laser Deposition (PLD). The films were protected by a gold film. The critical current density $\mathrm{j}_{\mathrm{c}}$ was measured as $2-5 \cdot 10^{6} \AA / \mathrm{cm}^{2}$. Both sapphire wafers were then repolished by a chemomechanical method [6] on their uncoated side for 45 minutes. To remove residual polishing solution we dipped the wafers in $\mathrm{KOH}$ and cleaned them in an ultrasonic bath for a few minutes. The wafers were positioned back-to-back in a microcleanroom setup. After rinse in deionised water to remove dust particles and drying by rotation, the wafers were contacted. After application of local pressure the bonding starts spontaneously and spreads over the whole wafer area. By the crack opening method [7] the initial bond energy was determined to be $300 \mathrm{~mJ} / \mathrm{m}^{2}$ after room temperature bonding. A thermal treatment of above $500^{\circ} \mathrm{C}$ for 10 hours enhanced the bond energy sufficiently to withstand quenching liquid nitrogen. Higher bond energies can be achieved by higher annealing temperatures (Fig. 2).

Transmission electron microscopy as shown in Fig. 3 reveals intimate contact of the two lattices in spite of some waviness of the interface. At lower magnification, the diffraction contrast exhibits nearly periodic strain fields probably due to dislocations that result from a remaining low crystallographic misorientation of the lattices of the wafers.

Measurements at liquid nitrogen yield a decrease of $j_{c}$ of about one order in magnitude due to the annealing in air. We suppose that annealing in an oxygen atmosphere will 


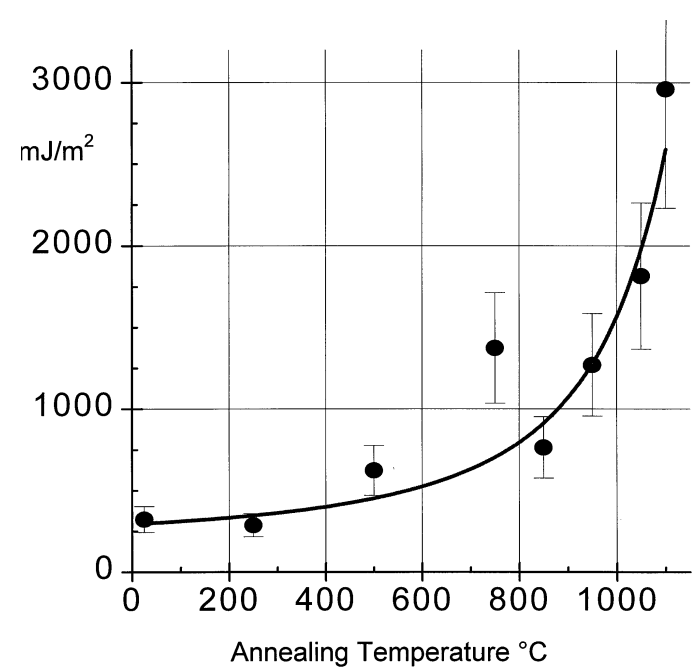

Fig. 2. Bond energy of directly bonded sapphire on sapphire $v s$ annealing temperature. The curve is a fit to the measured datapoints

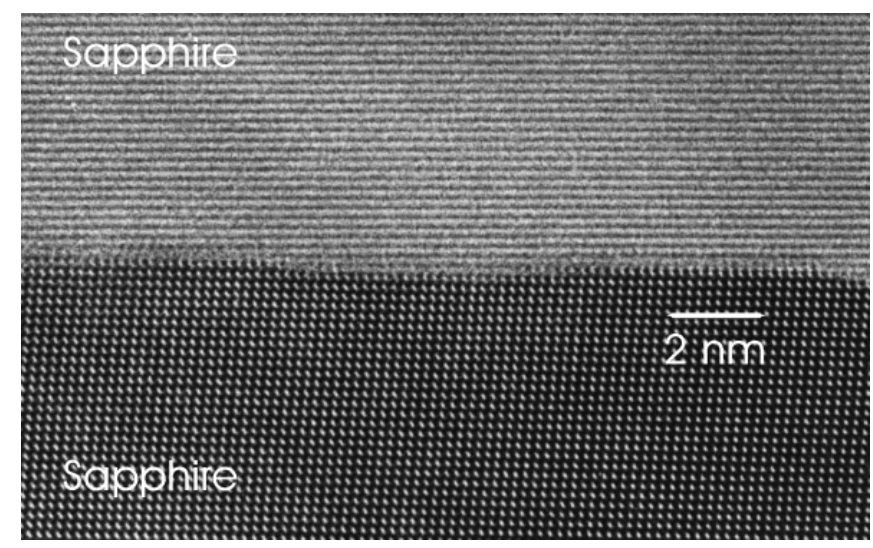

Fig. 3. TEM cross section of the sapphire/sapphire bonding interface annealed at $1100^{\circ} \mathrm{C}$ for $5 \mathrm{~h}$

prevent any degradation in the superconducting properties of our YBCO films. Results on annealing in oxygen will be published in the near future. Further investigations will concentrate on the electrical properties of the double side coated sapphire wafer in the desired range of high frequencies.

Let us finally mention that recent progress in back-toback wafer bonding has lead to an analogous proposal of double sided YBCO films on $\mathrm{LaAlO}_{3}$ substrates by wafer bonding [8].

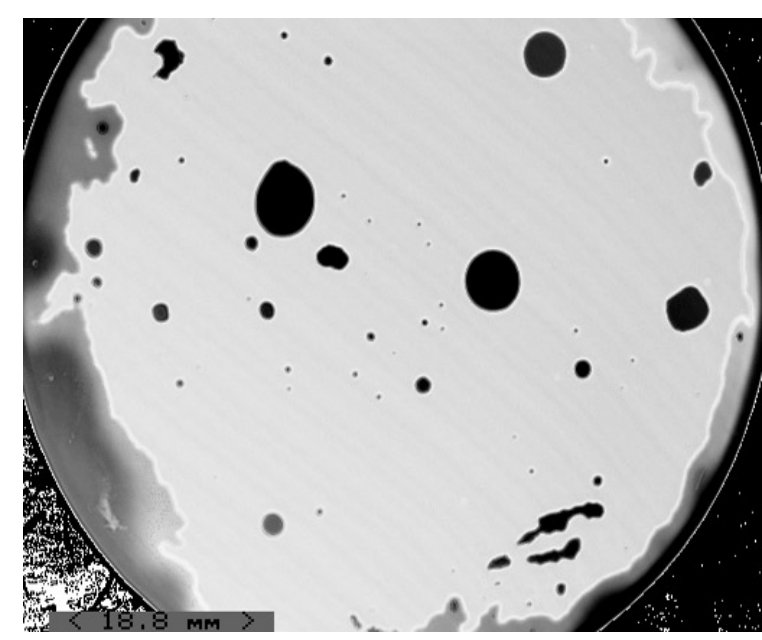

Fig. 4. Sound response of 3 inch $\mathrm{Au} / \mathrm{YBCO} / \mathrm{CeO}_{2}$ coated sapphire wafers directly bonded back-to-back on $\mathrm{Au} / \mathrm{YBCO} / \mathrm{CeO}_{2}$ coated sapphire

Acknowledgements. We acknowledge Dr. N.D. Zakharov for taking TEM lattice images. This work was funded by the BMBF (contract 13N6451/1).

\section{References}

1. Z.-Y. Shen: In High-Temperature Super-conducting Microwave Circuits, (Artech House, Inc., London 1994), p. 103

2. F. C. Wellstood, J. J. Kingston and J. Clarke: J. Appl. Phys. 75, 683 (1994)

3. P. Kopperschmidt, G. Kästner, D. Hesse und U. M. Gösele, Proc. 5. Statusseminar Supraleitung und Tieftemperaturphysik. VDI Technology Center, Cologne, (June 1996), in press

4. S. Bengtsson: J. Electronic Materials 21, 669 (1992)

5. C. Hunt, H. Baumgart, S. Iyer, U. Gösele, T. Abe (eds.): Proc. 3rd Int. Symp. Semiconductor Wafer Bonding: Science, Technology and Applications (Electrochem. Soc., Pennington, NJ 1995) Proc. Vol. 95-7

6. J. Haisma, G. A. C. M. Spierings, T. M. Michielsen, C. L. Adema: Philips. J. Res. 49, 23 (1995)

7. W. Maszara, G. Goetz, A. Caviglia, J. B. McKnitterick: J. Appl. Phys. 64, 2987 (1986)

8. C. B. Eom, L. Huang, R. A. Rao, Q. Y. Tong, U. Gösele: IEEE Trans. Appl. Superconductivity (August 1996), in press 